
ematics

\title{
Images segmentation using a modified Hopfield artificial neural network
}

\author{
Daniela O. Albanez ${ }^{1}$ \\ Postgraduate Program in Computer Science, UFU, Uberlândia, MG \\ Sérgio F. da Silva ${ }^{2}$ \\ Postgraduate Program in Modeling and Optimization, UFG-RC, Catalão, GO \\ Marcos A. Batista ${ }^{3}$ \\ Postgraduate Program in Modeling and Optimization, UFG-RC, Catalão, GO \\ Célia A. Zorzo Barcelos ${ }^{4}$ \\ Graduate Program in Computer Science, UFU, Uberlândia, MG
}

\begin{abstract}
Good image segmentation can be achieved by finding the optimum solution to an appropriate energy function. A Hopfield neural network has been shown to solve complex optimization problems fast, but it only guarantees convergence to a local minimum of the optimization function. This paper proposed a little modification in the Hopfield neural network, the experimental results were made with proposed model for gray-scale images segmentation on synthetic and satellite images showing its effectiveness.
\end{abstract}

Keywords. Image segmentation, Hopfield neural network, satellite images,

\section{Introduction}

The image segmentation is an important component of computer vision systems, whose goal is to partition a given image into meaningful regions and label each region by a region type. Also, it is a prerequisite for high-level image understanding and interpretation. A class per se is constituted by segmentation techniques adopting classification techniques based on neural networks.

It is well-known that the design of artificial neural networks (ANN's) try to imitate the information processing of biological neural cells. They offer two important properties in pattern recognition tasks: their extended parallel processing capability and nonlinear characteristics are used for classification and clustering, which allows for very fast computational times and makes them suitable for real time applications and good robustness to disturbances, which allows for reliable estimates. In the field of pattern recognition and decision making, ANN's have been established as a promising implementation of statistical, nonparametric, discriminant analysis because they can learn and synthesize the available information without requiring any statistical modeling of the problem [13].

ANN's are composed of many computational elements connected by links with variable weights. The complete network, therefore, represents a very complex set of inter-dependencies

\footnotetext{
${ }^{1}$ doalbanez6@gmail.com

${ }^{2}$ sergio@ufg.br

${ }^{3}$ marcos.batista@pq.cnpq.br

${ }^{4}$ celiazb@ufu.br
} 
which may incorporate any degree of non-linearity, allowing very general function to be modeled. Training time are usually very long, but after training, the classification using artificial neural networks is rapid. Usually the number of classes is derived with some a priori knowledge on the problem or in a pre-processing stage.

A number of algorithms have been proposed for segmenting gray-level images with neural networks [7]. Image segmentation may be considered as a clustering process in which the pixels are classified into the attributed regions based upon their gray-level values. In this paper, we will discuss a neural network based technique, Hopfield Neural Network (HNN), for images segmentation.

More and more researchers have sought knowledge to segmentation images using HNN, we can name some examples low:

Huang [6] and Tenorio [12] applied HNN to image segmentation and image matching.

Cheng [2] tackled the image segmentation problem by minimizing the cost function, which is defined as the mean distance measured between the gray level values and the member of classes.

Huang [6] and Campadelli [1] proposed a different network structure which used a $N \times$ $M \times S$ neuron array with the columns and the rows representing the $N \times M$ image pixels and the layers representing $\mathrm{S}$ classes of the object in the segmented image.

Rout [8] and Shen [11] used HNN to find an optimal threshold surface, which is determined by interpolating the image gray levels at edge points for segmenting image into different areas.

In this paper, we propose a small change in energy function as a result we apply the model proposed for segmentation on synthetics and satellite images, showing the effectiveness of the network.

The remainder of the paper is organized as follows: Section 2 reviews Hopfield Neural Network. Section 3 presents our proposed HNN model. Section 4 presents the implementation and results obtained with the proposed model. Finally, Section 5 presents the considerations.

\section{Hopfield Neural Network (HNN)}

Hopfield neural networks are artificial neural networks that have feedback and can be used as associative memories, in which the network is able to store information based some of its states. In addition, they can solve combinatorial optimization problems [3].

Hopfield [4] proposed a neural network architecture composed of neurons with graded responses (sigmoid input-output relation). He showed that the HNN can solve complex optimization problems such as a traveling salesman problem [5] fast once a proper energy function has been chosen. As Hopfield neurons are similary the real biological neurons, they can be implemented by real physical devices. It should be noted, however, that a HNN can solve optimization problems fast when a proper energy function is given, but it only guarantees convergence to a local minimum, not to the global minimum solution.

HNN for the optimization application consist of many interconnected neuron elements. The HNN structure consist of $N \times M$ neurons with each row representing a pixel and class column representing a cluster. The network classify the image of the $N$ pixels of the $P$ features among $M$ class such that the assignment of the pixels minimizes the criterion function [9]:

$$
E=\frac{1}{2} \sum_{k=1}^{N} \sum_{l=1}^{M} R_{k l}^{2} V_{k l}^{2}+c(t) \sum_{k=1}^{N} \sum_{l=1}^{M} N_{k l} V_{k l}
$$


where $R_{k l}$ is the Mahalanobis distance measure between the $k$ th pixel and the centroid of the class $l, V_{k l}$ is the output of the $k l$ th neuron, $N_{k l}$ is an $N \times M$ vector of independent high frequency white noise source, $c(t)$ is a parameter controlling the magnitude of noise that must be selected in such a way that it approaches zero as time $t$ tends to "infinity" and can be mathematically as [10]

$$
c(t)=\beta e^{-\alpha t}
$$

where $\beta>0$ controls the initial amplitude of the noise at $(t=0)$ and $\alpha>0$ determines the rate of noise damping. The minimization is achieved by solving a set of motion equations satisfying [9]

$$
\frac{\partial U_{k l}}{\partial t}=-\mu(t) \frac{\partial E}{\partial V_{k l}}
$$

where $U_{k l}$ is a input of the $k$ th neuron and $\mu(t)$ is a scalar positive function of time, is define by $[9]$ :

$$
\mu(t)=t\left(T_{s}-t\right)
$$

where $t$ is the iteration step and $T_{s}$ is the maximum value of interactions. If the input-output function of the neuron is increasing and the system satisfies (3), the energy continuously reduces as a function of time and the system converges to a local minimum.

\section{Proposed HNN Model}

Our first proposed concerns the formulation of the energy function of the segmentation process. We consider the classification as a partion of $N$ pixels of $P$ features into the best $M$ classes, and we propose the following equation

$$
E=\frac{1}{2} \sum_{k=1}^{N} \sum_{l=1}^{M} R_{k l}^{2} V_{k l}^{2}-\sum_{k=1}^{N} \sum_{l=1}^{M} U_{k l} V_{k l}
$$

where $U_{k l}$ is the external input, $R_{k l}$ is the Euclidean distance measure between the $k$ th pixel and the centroid of the class $l$ and $V_{k l}$ is the output of the $k l$ th neuron.

The second term of Equation 1 was modified and the term $c(t)=1$.

As a second contribution we replace the Mahalanobis distance by Euclidean distance. The generalized distance measure between the $k$ th pixel and the centroid of class $l$ by

$$
R_{k l}=\left\|X_{k}-\bar{X}_{l}\right\|_{A_{l}^{-1}} ; 1 \leq k \leq N, 1 \leq l \leq M
$$

where $X_{k}$ is a $\mathrm{P}$-dimensional feature vector of the $k$ th pixel $(\mathrm{P}=3$ for respect to the $\mathrm{RGB}$ color space components), $A_{l}$ is an identity matrix, $\bar{X}_{l}$ is the P-dimensional centroid vector of class $l$.

\section{Implementation and Experimental Results}

A large number of experiments have been conducted to observe the difference in the each network. The neural network architecture consists of a grid $N \times M$ neurons with each column 
representing a cluster and each row representing a pixel. The network is designed to classify the feature space without teaching through the compactness of each cluster calculed using the Euclidean distance measure by Equation 6 . By applying 3 to 5, we get a set of equations for neural dynamics given by

$$
\frac{d U_{k l}}{d t}-\mu(t)\left[R_{k l}^{2} V_{k l}-U_{k l}\right]
$$

where $U_{k l}$ and $V_{k l}$ are the input and output of the $k l$ th neuron, respectively. The input-output of $k$ th row is given by

$$
\begin{cases}V_{k l}(t+1)=1 & \text { if } U_{k l}<\operatorname{Max}\left[U_{k l}\right] \\ V_{k l}(t+1)=0 & \text { otherwise }\end{cases}
$$

where $\operatorname{Max}\left[U_{k l}\right]=0.0002$.

To facilitate the comprehension of the proposed PCNN model, we describe the process through the Algorithm 1.

\section{Algorithm 1: The proposed HNN image segmentation}

1) Initialize the input of neurons to random values (which amounts to a random assignment of $N$ pixels to $M$ classes).

2) Apply the following inputoutput relation given in 8 to obtain the new output values for each neuron, establishing the assignment of pixels to classes.

3) Compute the centroid $\bar{X}_{l}$ as

$$
\bar{X}_{l}=\left[\sum_{k=1}^{N} X_{k} V_{k l}\right] / n_{l}
$$

4) Update the inputs of each neuron by solving the set of differential equations in Eq. 3 using Euler's approximation

$$
U_{k l}(t+1)=U_{k l}+d U_{k l} / d t .
$$

5) If there is change in the inputs, repeat from step 2), else terminate.

\subsection{Experimental Results}

In this section, we presented the experimental results of proposed model for synthetics and satellite images segmentation. The first experiment is performed on synthetic images to demonstrate the effectiveness of the proposed model to separate the object and the background (see Fig. 1 for an example of these images). The second experiment is performed on satellite images for Goiás region, as shown in Fig. 2. The satellite images were obtained by satellite CBERS-2B (China-Bazil Earth Resources Satellite), available on the website of INPE (National Institute of Space Research). The objective was to segment each image in objects and the background, so, we apply two clusters HNN segmentation. The first column shows the original images. The second column shows the segmentation result using the proposed HNN model. 

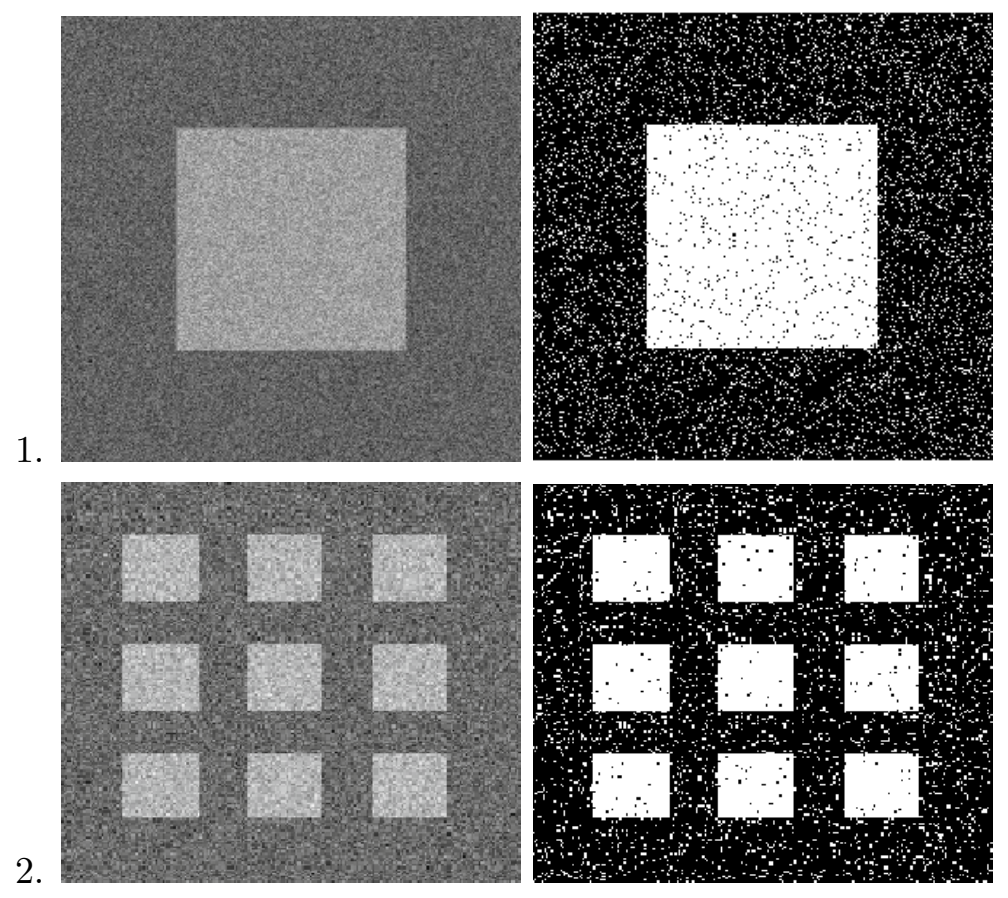

Figure 1: Experiments with synthetics images, in the left column shows the original image, in the right column shows the result of our algorithm HNN.

The proposed method is also applied on satellite images which contains water and agriculture area. In Fig. 2 experiment 1 shows reservoir and dam between the municipalities of Cascallho Rico and Três Ranchos, in experiment 2 shows the river Paranaíba in the state of Goiás in Brazil, in experiment 3 and 4 shows the rivers and agriculture areas, respectively.

As can be seen from the presented result, the HNN is giving good results in all the images. Remembering that, the objective was to segment each image in objects and the background, so, we apply two clusters HNN segmentation. With this, we observed that in satellite images this difference is very clear, being able to easily distinguish rivers and agricultural areas.

\section{Conclusions}

In this study we have presented some contributions in the use of a Hopfield network for the segmentation the synthectis and satellite images. In the proposed model, energy function of the original model was simplified, and the experiments on synthetic and satellite images demonstrated the efficiency of the network, and successful segmentation results were obtained. The satellite images of Goiás region were obtained by satellite CBERS-2B and using HNN algorithm with two clusters. We have shown that the performance of the network is mainly dictated by energy function optimization. For simplicity, the optimization criterion we considered the Euclidean distance between the pixel and the centroid of class. Also, the elimination of a noise term which allowed a good segmentation. 


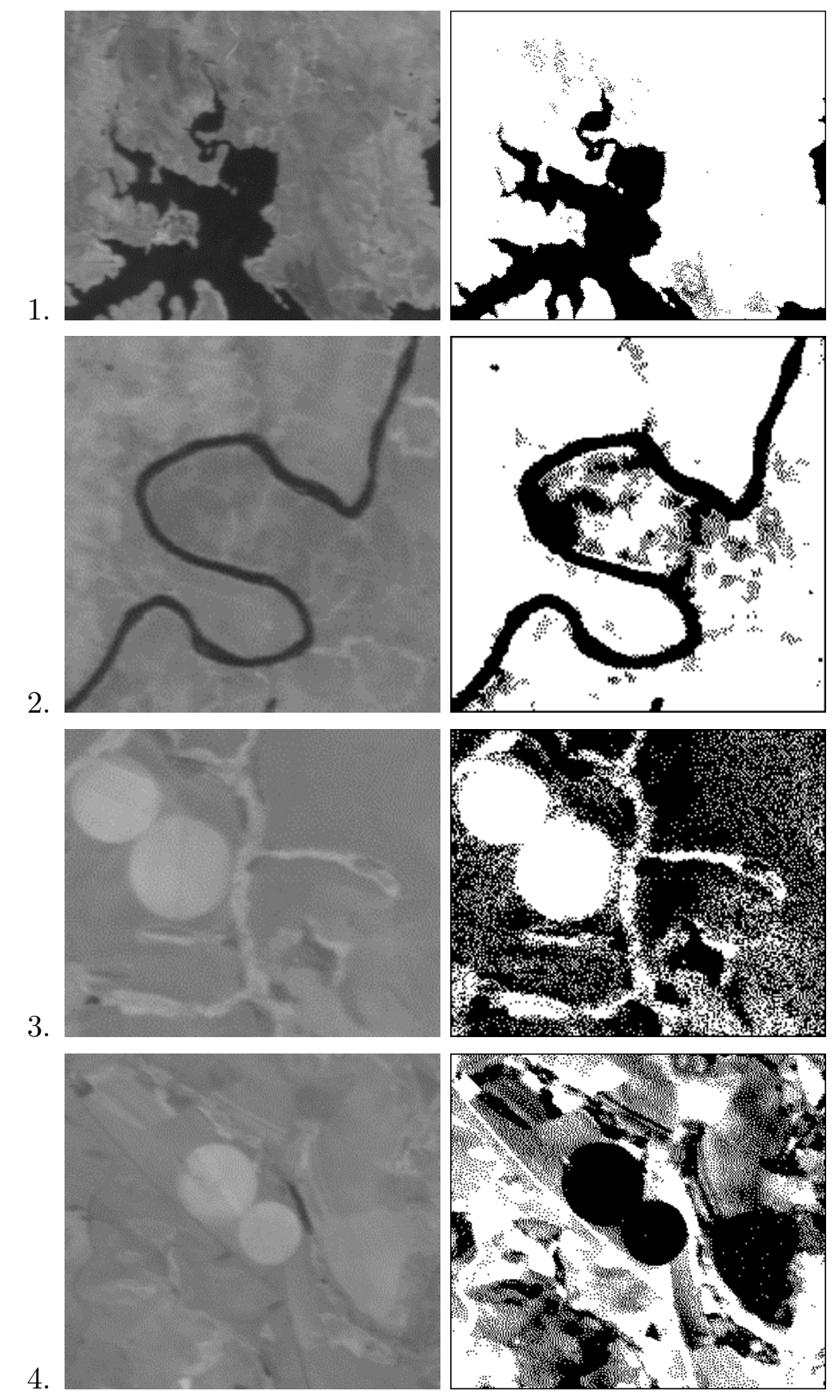

Figure 2: Experiments with satellite images, in the left column shows the original image, in the right column shows the result of our algorithm HNN. 


\section{Acknowledgments}

The authors would like to thank the Coordination of Higher Education Personnel Training (Capes), Brazilian National Council for Scientific and Technological Development (CNPq) and Research Support Foundation of Goiás State (FAPEG) for the financial support.

\section{References}

[1] P. Campadelli, D. Medici and R. Schettini. Color image segmentation using Hopfield networks, Image and Vision Contputing, 15(3):161-166, 1997.DOI:0.1016/S02628856(96)01121-3

[2] K. S. Cheng, J. S. Lin and C. W. Mao. The application of competitive Hopfield neural network to medical image segmentation, IEEE Transactions on Medical Imaging 15(4):560-567, 1996. DOI:10.1109/42.511759.

[3] S. Haykin. Redes Neurais: princípios e prática. Bookman, Porto Alegre, 2001.

[4] J. J. Hopfield. Neurons with Graded Response have Collective Computational Properties like Those of Two-state Neurons, Proc. Nail. Acad. Sci. USA, 81:3088-3092, 1984. DOI:10.1073/pnas.81.10.3088.

[5] J. J. Hopfield and D. W. Tank. Neural computation of decisions in optimization problems, Biological Cybernetics, 52:141-152, 1985. DOI: 10.1007/BF00339943.

[6] Chung-Lin Huang. Parallel image segmentation using modified Hopfield model, Pattern Recognition Letters, 13(5):345-353, 1992. DOI:10.1016/0167-8655(92)90032-U.

[7] N.R. Pal and S.K. Pal. A Review on Image Segmentation Techniques, Pattern Recognition, 26(9):1277-1294, 1993. DOI:10.1016/0031-3203(93)90135-J

[8] S. Rout, P. S. Seethalakshmy and J. Majumdar. Multi-modal image segmentation using a modified Hopfield neural network, Pattern Recognition, 31(6):743-750, 1998. DOI:10.1016/S0031-3203(97)00089-7.

[9] R. Sammouda, N. Adgada, A. Touir and A. Al-Ghamdi. Agriculture satellite image segmentation using a modified artificial Hopfield neural network, Computers in Human Behavior, 30:436-441, 2014. DOI:10.1016/j.chb.2013.06.025.

[10] R. Sammouda, N. Niki and H. Nishitani. A comparison of Hopfield Neural Network and Boltzmann Machine in Segmenting MR Images of the Brain, IEEE Transaction on nuclear science, 43(6):3361-3369, 1996. DOI:10.1109/23.552753.

[11] D. Shen and H. S. Horace. A Hopfield neural network for adaptive image segmentation: An active surface paradigm, Pattem Recognirion Letters, 18(1):37-48, 1997. DOI:10.1016/S0167-8655(96)00117-1.

[12] M.P. Tenorio. Real time noisy image segmentation using an artificial neural network model, IEEE First Internat. Conf. on Neural Network, 4:357-363, 1987.

[13] G.D. Tourassi and C.E. Floyd. Lesion size quantification in SPECT using an artificial neural network classification approach, Computers Biomed. Res., 28:257-270, 1995. 